\title{
Một số kỹ thuật phát hiện dị thường trên ảnh UAV ứng dụng trong công tác tìm kiếm cứu nạn
}

\author{
Nguyễn Văn Phương, Đào Khánh Hoài \\ Trường Đại học Kỹ thuật Lê Quý Đôn \\ Tác giả liên hệ: Nguyễn Văn Phương, phuongnv.dl@gmail.com \\ Ngày nhận: 19/09/2017, ngày sửa chữa: 14/06/2018, ngày duyệt đăng: 20/07/2018 \\ Xem sớm trực tuyến: 08/11/2018, định danh DOI: 10.32913/rd-ict.vol1.no39.581 \\ Biên tập lĩnh vực điều phối phản biện và quyết định nhận đăng: TS. Bùi Quang Hưng
}

Tóm tắt: Kỹ thuật phát hiện dị thường trên ảnh đa phổ và sieu phổ đã dành được nhiều quan tâm của cộng đồng xử lý ảnh viễn thám. Đã có nhiều nghiên cứu phát hiện và khảo sát tính hiệu quả của các kỹ thuật phát hiện dị thường trên một số loại ảnh vệ tinh siêu phổ và đa phổ. Trong thời gian gần đây dữ liệu ảnh của thiết bị bay không người lái (UAV) đã trở thành nguồn dữ liệu quan trọng phục vụ công tác tìm kiếm cứu nạn cả trên biển và đất liền. Đây là giải pháp công nghệ hiện đại và rất phù hợp với đặc thù của công tác này. Các kỹ thuật nhận dạng đối tượng cần tìm kiếm trên ảnh UAV bằng các thuật toán nhận dạng hình học chưa bao hàm hết đặc thù của các dấu hiệu tìm kiếm cứu nạn. Trong nghiên cứu này, nhóm tác giả kiểm chứng hiệu quả phát hiện dị thường trên các không gian màu khác nhau của ảnh UAV để phát hiện các tín hiệu cần tìm kiếm dựa vào toán tử $\mathrm{RX}$ và một số biến thể của $\mathrm{RX}$. Cách tiếp cận đề xuất được kiểm chứng thực nghiệm trên các bộ dữ liệu mẫu cho kết quả khả quan.

Từ khóa: Phát hiện dị thường, ảnh UAV, tìm kiếm cúu nạn.

Title: Anomaly Detection Techniques on UAV Images for Search and Rescue

Abstract: Anomaly detection techniques on multispectral and hyperspectral images have attracted many researchers in the remote sensing image processing community. Recently, unmanned aerial vehicles (UAVs) and UAV images have respectively become a technology and a data source for search and rescue. Geometrical pattern recognition techniques based purely on geometric features do not take into account many characteristics that are specific to search and rescue signatures. In this research, we investigated the effectiveness of the RX operator and its modifications on different color spaces of UAV images of different terrain types. The proposed approach was validated on sample images with positive results.

Keywords: Anomaly detection, UAV images, search and rescue.

\section{MỞ ĐẦेU}

Trước sự phát triển mạnh mẽ và ngày càng được ứng dụng phổ biến cả trong dân sự và quân sự của các thiết bị bay không người lái (UAV) trong những năm gần đây, UAV đã thực sự là một nguồn lực rất lớn cho sứ mệnh tìm kiếm cứu nạn bởi chúng có thể mang các thiết bị thu thập hình ảnh có độ phân giải cao, có thể hoạt động trong phạm vi rộng lớn với địa hình đa dạng mà không cần quá nhiều nhân lực và chi phí cho quá trình tìm kiếm. Nhưng với số lượng lớn ảnh có thể thu thập được, vấn đề phát sinh là xử lý, đánh giá và xác định các đối tượng cần quan tâm khi tìm kiếm cứu nạn như người, vật thể hoặc các chi tiết khác trong những ảnh này như thế nào? Nếu công việc này được thực hiện chủ yếu bằng thủ công, sẽ tốn khá nhiều thời gian và không đảm bảo độ tin cậy, làm giảm khả năng sống sót của nạn nhân. Các kỹ thuật tự động phát hiện dị thường màu trên ảnh có thể hỗ trợ đẩy nhanh quá trình tìm kiếm cứu nạn. Dị thường màu có thể được xem là một điểm, hoặc một cụm các điểm ảnh có sự khác biệt đáng kể về màu sắc so với các điểm ảnh lân cận trên ảnh UAV.

Hầu hết các nghiên cứu trong lĩnh vực phát hiện dị thường tập trung vào ảnh đa phổ hoặc ảnh siêu phổ (sử dụng trong hình ảnh y tế và địa chất) [1]. Toán tử dò dị thường Reed-Xiaoli $(\mathrm{RX})$ đã được sử dụng rộng rãi và thường được coi là một chuẩn mực [2]. Toán tử này so sánh các điểm ảnh riêng lẻ với cửa sổ lân cận hoặc thống kê toàn cục theo giả định rằng dữ liệu quang phổ đa biến tuân theo phân bố Gauss [3]. Có rất nhiều biến thể của toán tử RX 
dựa trên cửa sổ cho phép thích nghi tốt hơn trên toàn bộ hình ảnh, chẳng hạn như $\mathrm{RX}$ cục bộ (local) và bán cục bộ (quasi-local) [4].

Trong nghiên cứu của Harsanyi [5], một biến thể của toán tử RX là UTD-RX được bổ sung để phát hiện các mục tiêu có xác suất thấp trên các khung cảnh trong ảnh có mô hình phân bố dữ liệu chưa rõ. Nghiên cứu của Chang và nhóm nghiên cứu [6] đã khảo sát tính hiệu quả của UTD$\mathrm{RX}$ và một số biến thể khác như $\mathrm{RX}$ chuẩn hóa (NRX: Normalized $\mathrm{RX}$ ) và $\mathrm{RX}$ cải tiến (MRX: Modified $\mathrm{RX}$ ) trên ảnh siêu phổ AVIRIS.

Trong một số trường hợp, khi số lượng điểm dị thường lớn, dữ liệu ảnh có phân bố yếu hoặc không rõ ràng, khi đó ma trận hiệp phương sai không phản ánh sát với phân bố nền và hệ quả là toán tử $\mathrm{RX}$ không phát huy tốt hiệu quả phát hiện dị thường. Để giải quyết tình huống này, nhóm nghiên cứu của Ren [7] đã đề xuất một biến thể khác của RX là RX có trọng số (WRX: Weighted RX).

Trong một số tài liệu nghiên cứu phục vụ cho công tác tìm kiếm cứu nạn, một cách tiếp cận của các phương pháp phân tích ảnh màu thu nhận từ các thiết bị bay là tăng độ bão hòa của màu hiếm và giảm độ bão hòa của các màu phổ biến để giúp người sử dụng xác định được các màu dị thường [8]. Các nghiên cứu gần đây đã khảo sát việc điều chỉnh một số kỹ thuật siêu phổ và nhận thấy rằng các toán tử dò tìm mục tiêu được mô tả ở trên có thể hỗ trợ được trong tìm kiếm cứu nạn và toán tử $\mathrm{RX}$ có ưu thế phát hiện hiệu quả hơn và ít phụ thuộc vào việc điều chỉnh tham số [3]. Vì vậy, trong nghiên cứu này, nhóm tác giả tập trung nghiên cứu khảo sát khả năng phát hiện dị thường trên ảnh màu RGB thu từ UAV trên các địa hình khác nhau phục vụ cho công tác tìm kiếm cứu nạn.

\section{THUẬT TOÁN RX VÀ CÁC BIẾN THỂ CỦA NÓ}

\section{Thuât toán $\mathbf{R X}$}

Thuật toán RX cơ bản là thuật toán chuẩn phát hiện dị thường, ban đầu được phát triển bởi Reed và Yu [3] cho các ảnh đa phổ. Thuật toán cơ bản được mô tả bao gồm hai giả thiết. Giả thiết thứ nhất, $H_{0}$ : ảnh nền tuân theo một phân phối chuẩn (phân phối Gauss) với trung bình không và ma trận hiệp phương sai chưa biết, $\mathcal{N}(0, K)$, được ước lượng từ dữ liệu toàn cục hoặc cục bộ. Giả thiết thứ hai, $H_{1}$ : mục tiêu là sự kết hợp tuyến tính của tín hiệu mục tiêu và nhiễu nền. Vì vậy, vector phổ được biểu diễn bởi một phân phối chuẩn (phân phối Gauss) với giá trị trung bình bằng tín hiệu mục tiêu (hoặc nhiều mục tiêu) và nhiễu cộng bằng ma trận hiệp phương sai nền trong giả thiết thứ nhất.

$$
\begin{aligned}
& H_{0}: x(n)=x^{0}(n), \\
& H_{1}: x(n)=x^{0}(n)+b s(n),
\end{aligned}
$$

trong đó, $s(n) \in S=[s(1), s(2), \ldots, s(N)]$ là một mẫu tín hiệu dạng vector hàng gồm $J$ phần tử, $x(n)=$ $\left[x_{1}(n), x_{2}(n), \ldots, x_{J}(n)\right]^{T}, n=1,2, \ldots, N$, đại diện cho $J$ ảnh phổ con tương quan $(N>J)$ có thể chứa tín hiệu quang học với hình dạng biết trước và vị trí không biết trước. $x^{0}(n)$ là vector nhiễu tạp dư, $b=\left[b_{1}, b_{2}, \ldots, b_{j}\right]^{T}$ là vector cường độ tín hiệu không biết trước gồm $j$ phần tử, $j=1,2, \ldots, J$. Khi đó, toán tử dò tìm dị thường của $\mathrm{RX}$ như sau:

$$
\delta_{R X}(r)=(r-\mu)^{T} K_{\mathrm{L} \times \mathrm{L}}^{-1}(r-\mu),
$$

trong đó, $r$ là vector phổ của điểm ảnh, $\mu=\frac{1}{N} \sum_{i=1}^{N} r_{i}(N$ là tổng số điểm ảnh) là vector phổ trung bình của khu vực quan tâm, $L$ là số kênh phổ, $K=\frac{1}{N} \sum_{i=1}^{N}\left(r_{i}-\mu\right)\left(r_{i}-\mu\right)^{T}$ là ma trận hiệp phương sai của các kênh phổ.

Quá trình dò tìm dị thường của $\mathrm{RX}$ dựa trên việc khai thác sự khác biệt giữa các điểm ảnh của tín hiệu phổ đầu vào và các điểm ảnh xung quanh. Công thức (2) cơ bản rất giống với công thức tính khoảng cách Mahalanobis.

Trong bài báo này, chúng tôi thực hiện hai phiên bản của thuật toán RX, đó là:

○ RX toàn cục (GRX: Global-RX): ma trận hiệp phương sai $K$ và vector phổ trung bình toàn cục $\mu$ được xác định từ toàn bộ hình ảnh và tất cả các dải phổ được áp dụng cho phương trình (2).

- $\mathrm{RX}$ địa phương (LRX: Local-RX): từng điểm ảnh được đánh giá một cách riêng lẻ, ma trận hiệp phương sai $K$ và vector phổ trung bình cục bộ $\mu$ được xác định cục bộ trong một cửa sổ xung quanh điểm ảnh đang xét.

\section{Các thuật toán $\mathrm{NRX}$ và $M R X$}

Thật thú vị khi thấy rằng phương trình (2) hoạt động theo kiểu bộ lọc khớp, hiệu quả của bộ lọc này phụ thuộc vào hai tham số: tín hiệu khớp (phù hợp) $(r-\mu)^{T} K_{L \times L}^{-1}$ và hằng số tỷ lệ $k(k=1$ trong công thức (2)). Với lợi thế này, có hai lựa chọn để thay thế $R X$ là $N R X$ và $M R X$. Hai thuật toán thay thế $\mathrm{RX}$ này được biểu diễn lần lượt theo công thức (3) và (4), được phát triển bằng cách thiết lập lần lượt $k=\left[(r-\mu)^{T}(r-\mu)\right]^{-1}$ và $k=\left[(r-\mu)^{T}(r-\mu)\right]^{-1 / 2}$ theo trình bày tại [6]:

$$
\begin{aligned}
& \delta_{N R X}(r)=\frac{(r-\mu)^{T} K_{L \times L}^{-1}(r-\mu)}{(r-\mu)^{T}(r-\mu)}, \\
& \delta_{N R X}(r)=\frac{(r-\mu)^{T} K_{L \times L}^{-1}(r-\mu)}{\sqrt{(r-\mu)^{T}(r-\mu)}} .
\end{aligned}
$$

\section{Thuật toán WRX}

Mục tiêu dị thường được coi là nhỏ và phần nền ảnh có tính đồng nhất, ma trận hiệp phương sai của toàn bộ ảnh có thể xem như là ma trận hiệp phương sai của tập mẫu 
ảnh nền. Ngoài ra, các mục tiêu dị thường hoặc mục tiêu nhỏ nhân tạo (do con người tạo ra) có thể được tách ra ở dạng các giá trị ngoại lai trên ảnh. Do đó, thuật toán RX sử dụng khoảng cách Mahalanobis để tìm mục tiêu dị thường bằng cách sử dụng ma trận hiệp phương sai của tập mẫu để làm trơn các điểm ảnh nền, sau đó các điểm ảnh dị thường được tách ra. Không thành vấn đề khi số lượng điểm ảnh dị thường là ít. Nhưng bởi vì thuật toán này giả định mô hình nhiễu Gauss và sử dụng ma trận hiệp phương sai của tập mẫu để làm trơn dữ liệu, nếu tỉ lệ số điểm dị thường trên tổng số điểm ảnh tương đối lớn, ma trận hiệp phương sai của tập mẫu có thể không đại diện cho phân phối nền. Trong trường hợp này, thuật toán RX sẽ hoạt động không tốt. Để giải quyết vấn đề này, Ren và nhóm nghiên cứu [7] đã đề xuất thuật toán phát hiện dị thường trên ảnh siêu phổ sử dụng ma trận hiệp phương sai có trọng số. Trọng số thích hợp được gán cho mỗi điểm ảnh trong ma trận hiệp phương sai của tập mẫu sử dụng khoảng cách của nó tới trung tâm của dữ liệu đại diện cho phân bố nền. Vì vậy, ma trận hiệp phương sai có trọng số có thể được viết lại thành:

$$
\begin{aligned}
K_{w} & =\frac{\sum_{i=1}^{N} w_{i}\left(r_{i}-\mu_{w}\right)\left(r_{i}-\mu_{w}\right)^{T}}{\sum_{i=1}^{N} w_{i}}, \\
\mu_{w} & =\frac{\sum_{i=1}^{N} q_{i} r_{i}}{\sum_{i=1}^{N} q_{i}}
\end{aligned}
$$

trong đó, $w_{i}$ và $q_{i}$ lần lượt là các trọng số vô hướng của các điểm trong ảnh, được mô tả như sau:

$$
\begin{aligned}
q_{i} & =\frac{1}{1+\left\|r_{i}-\mu\right\|}, \\
w_{i} & =\frac{1}{1+\left\|r_{i}-\mu_{w}\right\|} .
\end{aligned}
$$

Cuối cùng, bộ lọc $\mathrm{RX}$ với ma trận hiệp phương sai có trọng số có thể được áp dụng cho ảnh,

$$
\delta_{W R X}(r)=\left(r-\mu_{w}\right)^{T} K_{w}^{-1}\left(r-\mu_{w}\right) .
$$

\section{Thuật toán UTD}

Một kỹ thuật phát hiện dị thường được Harsanyi [5] phát triển, gọi là phát hiện mục tiêu xác suất thấp (LPTD: Low probability target detector), công thức như sau:

$$
\delta_{L P T D}(r)=1_{L \times L} R_{L \times L}^{-1} r .
$$

Kỹ thuật phát hiện được thiết kế dựa trên ma trận tương quan mẫu $R$. Nếu $R$ được thay thế bằng ma trận hiệp phương sai mẫu $K$ thì LPTD sử dụng ma trận hiệp phương sai mẫu $K$ được gọi là kỹ thuật phát hiện mục tiêu đồng nhất (UTD: Uniform target detector), công thức như sau:

$$
\delta_{U T D}(r)=\left(1_{L \times 1}-\mu\right)^{T} K_{L \times L}^{-1}(r-\mu),
$$

trong đó, $1_{L \times 1}$ là vector có kích thước $L$ với các phần tử đều là 1 . Vì vậy, các mục tiêu dị thường được giả định là tuân theo phân phối đều trên tất cả các kênh phổ. Do đó, UTD dự đoán để trích xuất tín hiệu nền, tín hiệu này có phân bố đều trong ảnh.

Bằng cách kết hợp UTD vào $\mathrm{RX}$ cơ bản, có thể loại bỏ nhiễu, cải thiện hiệu suất phát hiện của $\mathrm{RX}$ cơ bản. Ưu điểm này cho phép phát triển một thuật toán tìm kiếm dị thường mới bằng cách kết hợp giữa $\mathrm{RX}$ và UTD [6] như sau:

$$
\delta_{R X-U T D}(r)=(r-1)^{T} K_{L \times L}^{-1}(r-\mu) .
$$

\section{Thuật toán CRX}

Kể từ khi ra đời, thuật toán dò tìm $\mathrm{RX}$ bao gồm các phép tính vector trung bình và ma trận hiệp phương sai không thể thực hiện trong thời gian thực. Do đó, để thực hiện tính toán theo thời gian thực, một phiên bản của $\mathrm{RX}$ được đề xuất, sử dụng ma trận tương quan $R$ thay cho ma trận hiệp phương sai $K$. Phiên bản RX này được gọi là "nhân quả" (CRX: Causal RX) có nghĩa là các thông tin sử dụng cho xử lý dữ liệu tại mỗi điểm ảnh chỉ dựa trên các điểm ảnh đã được xử lý,

$$
R\left(r_{k}\right)=\frac{1}{k} \sum_{i=1}^{k} r_{i} r_{i}^{T}
$$

Ma trận tương quan mẫu $R\left(r_{k}\right)$ có thể được tính toán song song, do đó thuật toán tìm kiếm có thể thực hiện trong thời gian thực [6],

$$
\delta_{C R X}\left(r_{k}\right)=(r-1)^{T} R_{L \times L}^{-1}(r-\mu) .
$$

\section{PHƯƠNG PHÁP PHÁT HIỆN DI THƯỜNG TRÊN ẢNH UAV DỰA TRÊN SỰ KHÁC BIẸTT VỀ MÀU SẮC}

Phân tích ảnh UAV ứng dụng trong công tác tìm kiếm cứu nạn có thể được tổng quát hóa là công việc nhận dạng các dấu hiệu dị thường có liên quan đến các đối tượng cần tìm kiếm cứu nạn. Các dấu hiệu tìm kiếm rất đa dạng và trong nhiều trường hợp rất khác biệt, tách rời và thậm chí là không liên quan trực tiếp đến đối tượng cần tìm kiếm. Các dấu hiệu tìm kiếm còn phụ thuộc vào cả thời gian tiến hành tìm kiếm. Ví dụ, khi sử dụng ảnh hàng không hoặc ảnh vệ tinh để tìm kiếm máy bay rơi trên đất liền. Nếu ảnh chụp khu vực báo nạn trong khoảng thời gian ngắn sau tai nạn thì dấu hiệu tìm kiếm đầu tiên nảy ra trong đầu các nhà phân tích ảnh sẽ là các đám khói. Nếu ảnh chụp sau thời điểm báo nạn một khoảng thời gian dài hơn, dấu hiệu tìm kiếm có thể là các vệt đổ của thực vật do máy bay gây ra khi tiếp đất. Khi đối tượng tìm kiếm là con người thì dấu hiệu tìm kiếm trên ảnh hàng không cũng khá đa dạng. Độ phân giải không gian của ảnh UAV phổ biến ở dải $0,07-0,3 \mathrm{~m}$. Đối tượng vật lý con người thường không hiện diện trên ảnh để nhận dạng, nếu có thì do cấu trúc địa 
hình bề mặt trái đất, sự che khuất bởi các đối tượng khác, độ phân giải ảnh và tư thế ngẫu nhiên khi bị nạn, hình dạng đối tượng người trên ảnh hàng không thường không tuân theo một khuôn mẫu chuẩn mực nào thuận lợi cho việc nhận dạng hình học. Một gợi ý tiếp theo chính là các đồ vật đi kèm với đối tượng tìm kiếm. Tương tự như đối tượng người, cách tìm kiếm bằng nhận dạng hình học cũng sẽ gặp khó khăn do mẫu nhận dạng không chuẩn tắc. Một cách tiếp cận nữa có thể ứng dụng được để phân tích ảnh hàng không ứng dụng trong tìm kiếm cứu nạn là phân tích màu sắc các vật thể có liên quan hoặc đi kèm với đối tượng cần tìm kiếm. Nhiều vật thể đi kèm trên máy bay bị nạn có màu sắc khác biệt với màu nền xung quanh trên bề mặt, đặc biệt khi tai nạn xảy ra ở các khu vực rừng núi hay trên biển. Một câu hỏi tiếp theo được đặt ra là sự khác biệt về màu sắc thể hiện như thế nào trên ảnh màu ba kênh RGB thu từ máy ảnh quang học. Các thành phần màu sắc có ảnh hưởng như thế nào trong quá trình phân tích dị thường bằng thuật toán tự động. Theo cách tư duy này nhóm nghiên cứu tiến hành khảo sát và phân tích hiệu quả của các thuật toán phát hiện khác biệt màu dựa trên toán tử $R X$ và một số biến thể của toán tử $\mathrm{RX}$ trên các không gian màu $\mathrm{RGB}$, LAB, XYZ, HSL, HSB, CMYK, YUV và YCbCr.

\section{THỰC NGHIỆM PHÁT HIÊ̂N DI THƯờNG TRÊN TẬP ẢNH UAV}

\section{Cấy mẫu dị thường}

Dữ liệu ảnh hàng không thu trong các tình huống tìm kiếm cứu nạn thực tế ở Việt Nam còn rất hiếm và cơ bản là không được phát hành công khai. Để kiểm nghiệm kết quả nghiên cứu, chúng tôi đã tiếp cận theo cách cấy một số mẫu dị thường vào các ảnh chụp từ thiết bị UAV, những ảnh này được trích từ phần dữ liệu thực nghiệm của đề tài cấp nhà nước mã số VT-UD.04/16-20 thuộc chương trình KHCN vũ trụ, sao cho có một cái nhìn thực tế nhất. Những ảnh này được chụp trên ba dạng địa hình khác nhau; ảnh 1 được thể hiện trên Hình 1 , chụp ở địa hình đồng bằng bằng máy ảnh Canon IXUS 127 HS ở độ cao 190 m, độ phân giải mặt đất là $62,7 \mathrm{~mm} /$ điểm ảnh; ảnh 2 và ảnh 3 được thể hiện lần lượt trên Hình 2 và Hình 4 , chụp ở địa hình tương ứng là rừng thưa và vùng biển bằng máy ảnh Sony DSC-WX220 ở độ cao 200m, độ phân giải mặt đất là $63,4 \mathrm{~mm} /$ điểm ảnh. Mỗi ảnh đưa vào kiểm nghiệm ở trên có kích thước $1000 \times 1000$ điểm ảnh và được cấy 03 mẫu dị thường khác nhau tùy theo các địa hình khác nhau, quy trình cấy mẫu như sau đây.

Đối với ảnh 1 (xem Hình 1) và ảnh 2 (xem Hình 2), là hai mẫu ảnh chụp địa hình tương ứng là đồng bằng và rừng thưa, ba mẫu áo có màu sắc khác nhau nhưng không quá nổi bật được cấy vào các vị trí có màu sắc gần với ảnh nền.

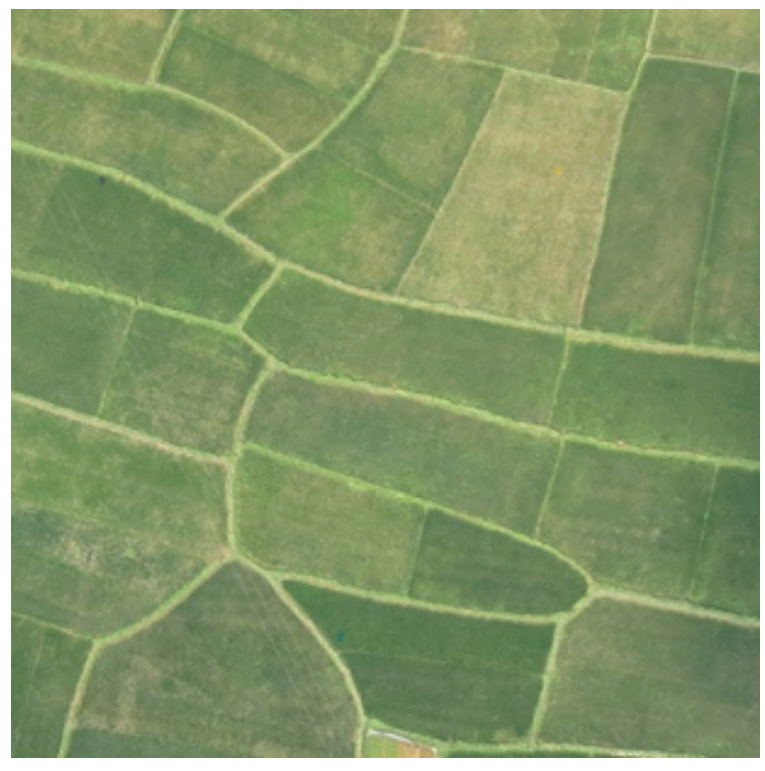

Hình 1 . Ảnh 1 chụp tai khu vực đồng bằng đã được cấy ba mẫu (mẫu 1 , mẫu 2 và mẫu 3 ).

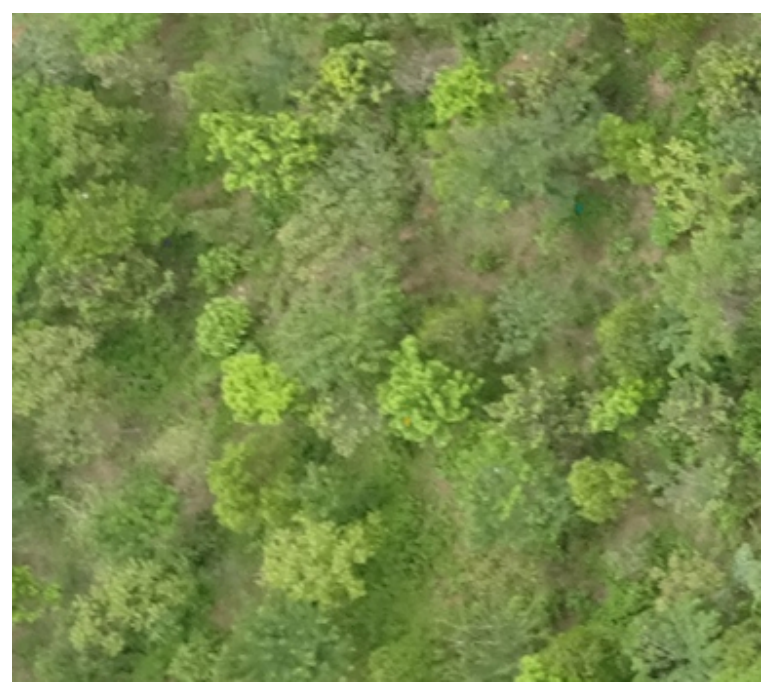

Hình 2. Ảnh 2 chụp tai khu vực rừng thưa đã được cấy ba mẫu (mẫu 1 , mẫu 2 và mẫu 3 ).

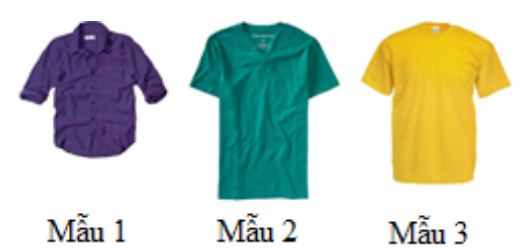

Hình 3. Ba mẫu dị thường dùng để cấy vào ảnh 1 và ảnh 2 .

Trong thực tế, mẫu 1 có kích thước $750 \times 683 \mathrm{~mm}$, mẫu 2 có kích thước $670 \times 787 \mathrm{~mm}$, mẫu 3 có kích thước $700 \times$ $750 \mathrm{~mm}$ (xem Hình 3). Khi cấy vào các ảnh, các mẫu này cũng phải thu nhỏ theo tỉ lệ tương đồng với độ phân giải 


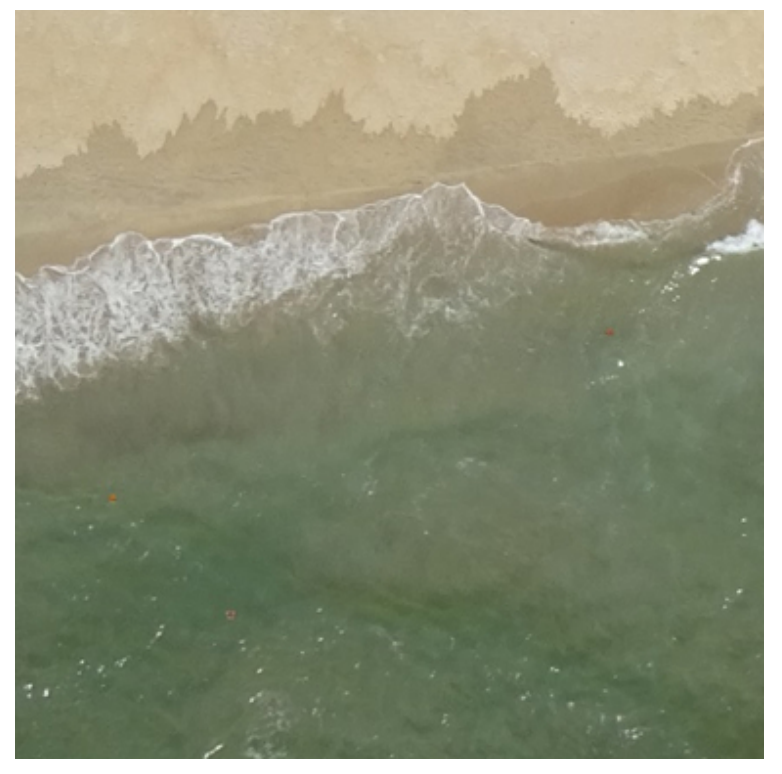

Hình 4. Ảnh 3 chụp tai khu vực vùng biển đã được cấy ba mẫu (mẫu 4 , mẫu 5 và mẫu 6 ).

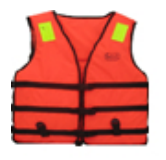

Mẫu 4

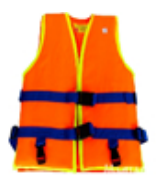

Mẫu 5

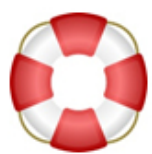

Mẫu 6
Hình 5. Ba mẫu dị thường dùng để cấy vào ảnh 3 .

của ảnh, cụ thể:

- Đối với ảnh 1, mẫu 1 kích thước co lại còn $12 \times 11$ điểm ảnh; mẫu 2 kích thước co lại là $11 \times 13$ điểm ảnh; mẫu 3 kích thước co lại là $11 \times 12$ điểm ảnh;

- Đối với ảnh 2 , mẫu 1 kích thước co lại là $12 \times 11$ điểm ảnh; mẫu 2 kích thước co lại là $11 \times 12$ điểm ảnh; mẫu 3 kích thước co lại là $11 \times 12$ điểm ảnh.

Đối với ảnh 3 (xem Hình 4), là ảnh chụp địa hình vùng biển, hai mẫu áo phao cứu sinh có màu sắc khác nhau và một mẫu phao cứu sinh được cấy ngẫu nhiên, chi tiết tại Hình 5.

Trong thực tế, mẫu 4 có kích thước $610 \times 530 \mathrm{~mm}$, mẫu 5 có kích thước $610 \times 708$ mm, mẫu 6 có kích thước $900 \times$ $900 \mathrm{~mm}$. Khi cấy vào ảnh 3 , các mẫu này cũng phải thu nhỏ theo tỉ lệ tương đồng với độ phân giải của ảnh, cụ thể: mẫu 4 kích thước co lại còn $10 \times 8$ điểm ảnh; mẫu 2 kích thước co lại là $10 \times 11$ điểm ảnh; mẫu 3 kích thước co lại là $14 \times 14$ điểm ảnh.

Để các mẫu giống tự nhiên hơn, chúng tôi đã tăng độ trong suốt của các mẫu lên $50 \%$ trước khi cấy vào. Điều này hoàn toàn phù hợp với tự nhiên là các mục tiêu thường bị lẫn vào các đối tượng lân cận.

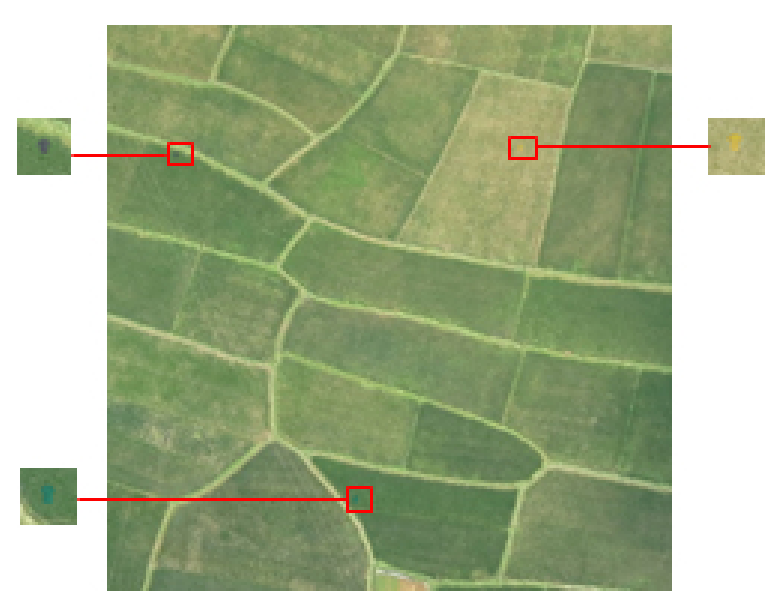

Hình 6. Các mẫu đã được cấy trên ảnh 1 .

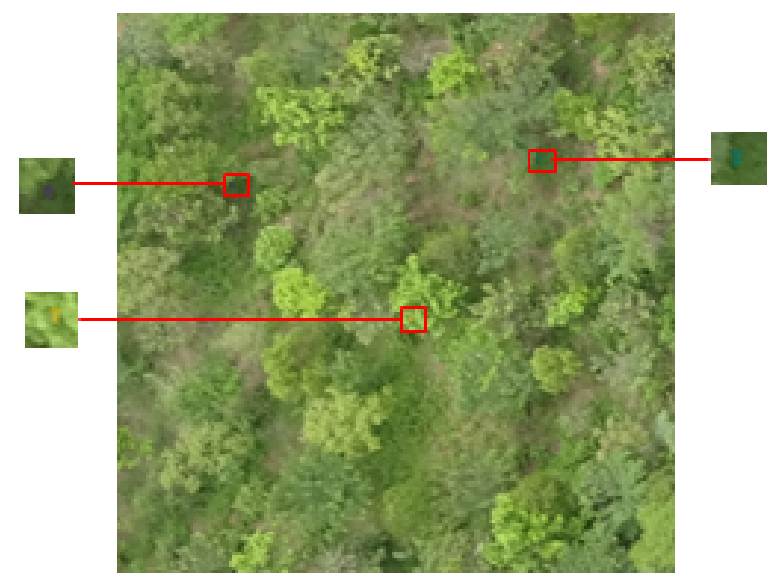

Hình 7. Các mẫu đã được cấy trên ảnh 2 .

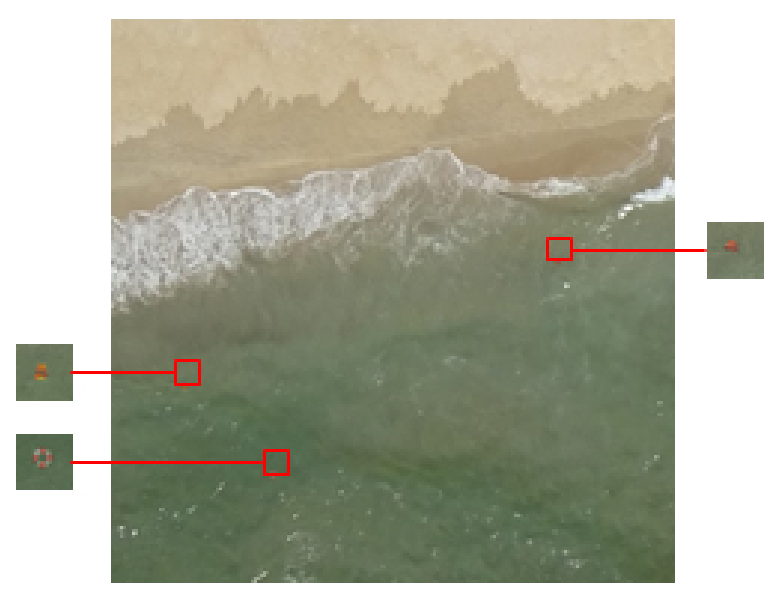

Hình 8. Các mẫu đã được cấy trên ảnh 3.

Trên các Hình 1,2 và 4 ta thấy rằng các mẫu dị thường sau khi được cấy lên ảnh cơ bản mắt thường khó có thể phát hiện được. Các Hình 6,7 và 8 là các hình ảnh phóng đại lên gấp ba lần so với các Hình 4,5 và 6 để minh họa các vị trí cấy mẫu. 
Bảng I

GIÁ TRI AUC TRUNG BÌNH CỦA TÁM THUẬT TOÁN ĐÁNH GIÁ CHO TÁM KHÔNG GIAN MÀU

\begin{tabular}{lllllllll}
\hline & GRX & LRX & NRX & MRX & WRX & UTD & RX-UTD & CRX \\
\hline RGB & $\mathbf{0 , 8 1 7}$ & 0,735 & 0,571 & 0,721 & 0,764 & 0,619 & 0,631 \\
LAB & $\mathbf{0 , 8 2 1}$ & 0,735 & 0,566 & 0,757 & 0,800 & 0,612 & 0,623 \\
XYZ & $\mathbf{0 , 7 4 6}$ & 0,708 & 0,559 & 0,672 & 0,675 & 0,645 & 0,649 \\
YCbCr & $\mathbf{0 , 8 1 7}$ & 0,736 & 0,557 & 0,716 & 0,787 & 0,446 & 0,371 \\
HSL & $\mathbf{0 , 7 4 7}$ & 0,704 & 0,475 & 0,526 & 0,720 & 0,528 & 0,365 \\
HSB & $\mathbf{0 , 7 5 5}$ & 0,701 & 0,472 & 0,574 & 0,720 & 0,520 & 0,595 \\
YUV & $\mathbf{0 , 8 1 7}$ & 0,735 & 0,555 & 0,719 & 0,794 & 0,475 & 0,552 \\
CMYK & 0,737 & 0,628 & 0,620 & 0,724 & $\mathbf{0 , 8 1 8}$ & 0,763 & 0,564 \\
\end{tabular}

Bảng II

GIÁ TRI AUC CỦA TÁM THUÂT TOÁN ĐÁNH GIÁ CHO TÁM KHÔNG GIAN MÀU TRÊN ẢNH NỀN KHU VỰC ĐỒNG BẰNG

\begin{tabular}{|c|c|c|c|c|c|c|c|c|}
\hline & GRX & LRX & NRX & MRX & WRX & UTD & RX-UTD & CRX \\
\hline RGB & 0,901 & 0,706 & 0,556 & 0,785 & 0,879 & 0,522 & 0,755 & 0,368 \\
\hline LAB & 0,902 & 0,707 & 0,588 & 0,856 & 0,887 & 0,524 & 0,730 & 0,431 \\
\hline XYZ & 0,781 & 0,702 & 0,526 & 0,741 & 0,758 & 0,598 & 0,743 & 0,391 \\
\hline $\mathrm{YCbCr}$ & 0,901 & 0,707 & 0,551 & 0,791 & 0,883 & 0,383 & 0,641 & 0,375 \\
\hline HSL & 0,887 & 0,705 & 0,484 & 0,479 & 0,945 & 0,678 & 0,516 & 0,644 \\
\hline HSB & 0,902 & 0,703 & 0,481 & 0,472 & 0,945 & 0,642 & 0,547 & 0,644 \\
\hline YUV & 0,901 & 0,706 & 0,544 & 0,791 & 0,888 & 0,463 & 0,585 & 0,379 \\
\hline CMYK & 0,727 & 0,617 & 0,577 & 0,732 & 0,935 & 0,823 & 0,167 & 0,763 \\
\hline
\end{tabular}

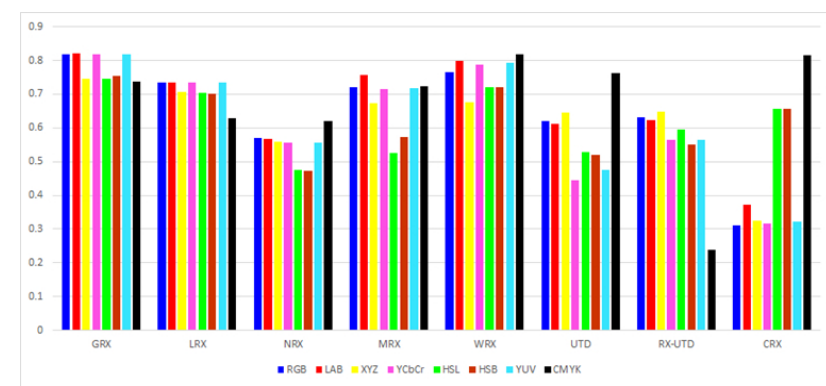

Hình 9. Giá trị AUC của tám thuật toán đánh giá cho tám không gian màu.

Sử dụng tám thuật toán đã nêu ở trong Mục II (GRX, LRX, NRX, MRX, WRX, UTD, RX-UTD, CRX) để đánh giá hiệu quả phát hiện dị thường trên tám không gian màu khác nhau (RGB, LAB, XYZ, YcbCr, HSL, HSB, YUV, CMYK), hiệu suất của mỗi thuật toán trên các không gian màu khác nhau được thể hiện thông qua đường cong đặc trưng hoạt động phí thu (ROC: Receiver operating characteristic). Diện tích dưới đường cong ROC (AUC: Area under the ROC curve) được sử dụng làm thước đo so sánh hiệu suất phát hiện dị thường giữa các thuật toán trên các không gian màu. Đầu vào của mỗi thuật toán là các ảnh đã cấy mẫu nêu ở trên, các ảnh này được biến đổi về các không gian màu khác nhau để đánh giá những ưu điểm và hạn chế. Bằng cách này, nhóm tác giả kỳ vọng sẽ đưa ra thuật toán phát hiện dị thường hoạt động trên các không gian màu phù hợp với từng địa hình hỗ trợ công tác tìm kiếm cứu nạn.

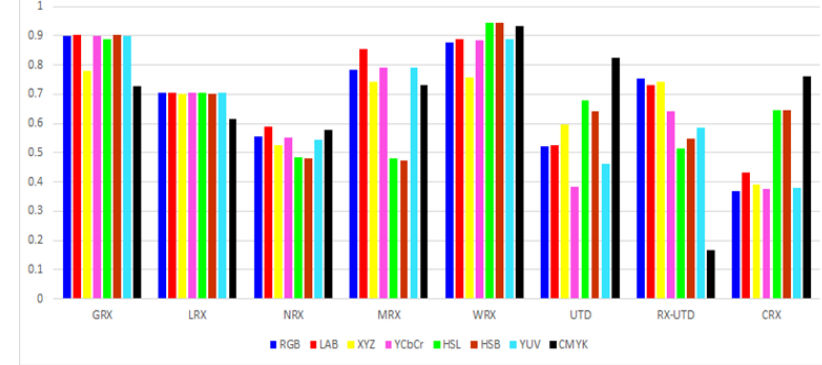

Hình 10. Giá trị AUC của tám thuật toán đánh giá cho tám không gian màu trên ảnh nền khu vực đồng bằng.

\section{Thuật toán}

Bảng I và Hình 9 so sánh giá trị AUC cho tám thuật toán trên tám không gian màu, hiệu suất này dựa trên kêt quả của các thuật chạy trên ba ảnh đầu vào với các dị thường được cấy vào như đã nêu ở trên. Trên Bảng I, các giá trị AUC này được tính trên cả ba ảnh sau đó lấy giá trị trung bình.

Bảng I và Hình 9 cho thấy, thuật toán GRX cho hiệu suất phát hiện dị thường tốt nhất trên không gian màu $\mathrm{LAB}$ và thuật toán này cũng cho thấy sự hiệu quả trên các không gian màu khác nhau, dù chuyển đổi từ không gian màu RGB sang các không gian màu khác nhưng hiệu suất phát hiện dị thường ít bị ảnh hưởng. Tiếp theo là thuật toán WRX và $C R X$, thuật toán WRX cũng cho hiệu suất tương đối ổn định trên các không gian màu khác nhau nhưng thuật toán CRX chỉ cho hiệu suất tốt nhất trên không gian màu CMYK, còn với các không gian màu khác cho hiệu suất thấp. Thuật toán NRX là thuật toán kém hiệu quả nhất. 
Bảng III

GIÁ TRI AUC CỦA TÁM THUẬT TOÁN ĐÁNH GIÁ CHO TÁM KHÔNG GIAN MÀU TRÊN ẢNH NỀN KHU VỰC RỪNG NÚI

\begin{tabular}{lllllllll}
\hline & GRX & LRX & NRX & MRX & WRX & UTD & RX-UTD & CRX \\
\hline RGB & $\mathbf{0 , 8 9 1}$ & 0,809 & 0,519 & 0,736 & 0,890 & 0,669 & 0,565 & 0,276 \\
LAB & 0,909 & 0,809 & 0,489 & 0,807 & $\mathbf{0 , 9 1 7}$ & 0,645 & 0,580 & 0,326 \\
XYZ & 0,813 & 0,730 & 0,527 & 0,633 & $\mathbf{0 , 8 3 0}$ & 0,678 & 0,599 & 0,287 \\
YCbCr & 0,891 & 0,810 & 0,484 & 0,719 & $\mathbf{0 , 9 0 4}$ & 0,324 & 0,687 & 0.289 \\
HSL & 0,688 & 0,717 & 0,440 & 0,592 & 0,626 & 0,317 & 0,691 & $\mathbf{0 , 7 7 2}$ \\
HSB & 0,693 & 0,710 & 0,429 & 0,640 & 0,626 & 0,315 & 0,691 & $\mathbf{0 , 7 7 2}$ \\
YUV & 0,891 & 0,809 & 0,487 & 0,725 & $\mathbf{0 , 9 0 4}$ & 0,328 & 0,739 & 0,291 \\
CMYK & 0,811 & 0,566 & 0,615 & 0,788 & $\mathbf{0 , 9 1 2}$ & 0,835 & 0,177 & 0,828 \\
\hline
\end{tabular}

Bảng IV

GIÁ TRI AUC CỦA TÁM THUÂT TOÁN ĐÁNH GIÁ CHO TÁM KHÔNG GIAN MÀU TRÊN ẢNH NỀN KHU VƯC BIỂN

\begin{tabular}{lllllllll}
\hline & GRX & LRX & NRX & MRX & WRX & UTD & RX-UTD & CRX \\
\hline RGB & 0,659 & $\mathbf{0 , 6 9 1}$ & 0,639 & 0,641 & 0,524 & 0,667 & 0,572 & 0,293 \\
LAB & 0,651 & $\mathbf{0 , 6 8 9}$ & 0,622 & 0,609 & 0,596 & 0,668 & 0,558 & 0,357 \\
XYZ & 0,645 & $\mathbf{0 , 6 9 0}$ & 0,625 & 0,643 & 0,436 & 0,659 & 0,604 & 0,297 \\
YCbCr & 0,659 & $\mathbf{0 , 6 9 1}$ & 0,637 & 0,639 & 0,574 & 0,631 & 0,366 & 0,286 \\
HSL & 0,667 & $\mathbf{0 , 6 8 9}$ & 0,501 & 0,507 & 0,589 & 0,590 & 0,578 & 0,553 \\
HSB & 0,670 & $\mathbf{0 , 6 9 1}$ & 0,505 & 0,609 & 0,589 & 0,604 & 0,418 & 0,553 \\
YUV & 0,659 & $\mathbf{0 , 6 9 1}$ & 0,635 & 0,642 & 0,589 & 0,633 & 0,367 & 0,296 \\
CMYK & 0,672 & 0,702 & 0,668 & 0,651 & 0,606 & 0,632 & 0,373 & $\mathbf{0 , 8 5 7}$ \\
\hline
\end{tabular}

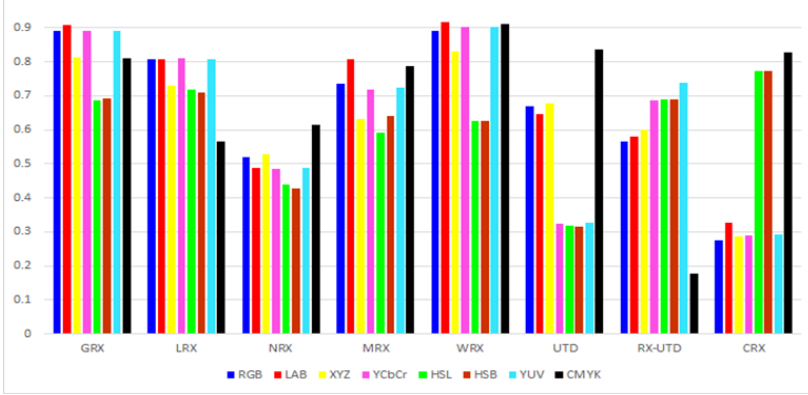

Hình 11. Giá trị AUC của tám thuật toán đánh giá cho tám không gian màu trên ảnh nền khu vực rừng núi.

Hiệu suất phát hiện dị thường của các thuật toán trên từng loại địa hình được thử nghiệm và phân tích cụ thể dưới đây.

Bảng II và Hình 10 cho thấy, thuật toán WRX cho hiệu suất phát hiện dị thường tốt nhất, sau đó đến thuật toán GRX. Thuật toán yếu nhất vẫn là NRX.

Bảng III và Hình 11 cho thấy, hiệu suất phát hiện dị thường của tốt nhất vẫn là thuật toán WRX, sau đó đến thuật toán GRX. Thuật toán yếu nhất vẫn là NRX.

Bảng IV và Hình 12 cho thấy, thuật toán WRX không còn chiếm ưu thế về hiệu suất phát hiện nữa, lúc này thuật toán CRX cho hiệu suất tốt nhất, sau đó đến thuật toán LRX, UTD và GRX. Thuật toán yếu nhất lại là RX-UTD

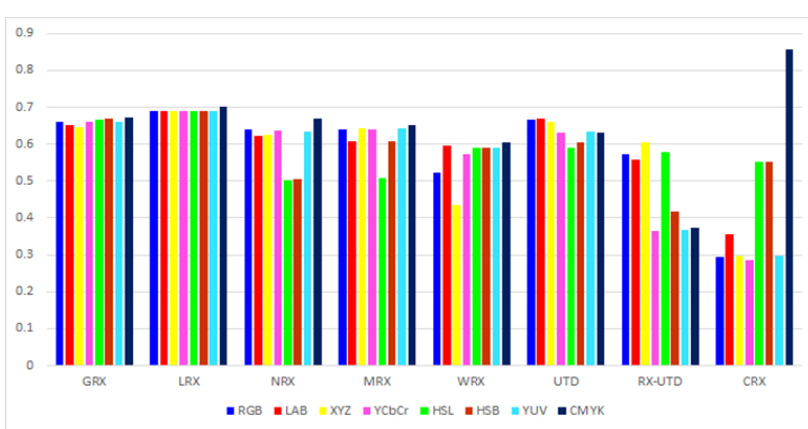

Hình 12. Giá trị AUC của tám thuật toán đánh giá cho tám không gian màu trên ảnh nền khu vực biển.

và WRX. Hiệu suất phát hiện dị thường của các thuật toán trên nền địa hình biển đã kém đi rất nhiều so với khi thực hiện trên địa hình đồng bằng.

Từ dữ liệu thu được ta thấy rằng, ở những địa hình càng phức tạp thì khả năng phát hiện dị thường của các thuật toán càng thấp, điều này hoàn toàn phù hợp với thực tế.

\section{Không gian màu}

Hình 9 cho thấy kết quả phát hiện dị thường của tám thuật toán thực hiện trên tám không gian màu khác nhau. Trên không gian màu RGB, mặc dù không cho hiệu quả tốt nhất với tất cả các thuật toán trên cả ba loại địa hình 
nhưng đây là không gian màu đáng tin cậy để thực hiện các thuật toán.

Trên không gian màu LAB, các thuật toán MRX trên địa hình đồng bằng và $\mathrm{WRX}$ trên địa hình rừng núi cho kết quả tốt nhất. Xét về tổng thể, không gian màu LAB tương đối ổn định và là không gian màu đáng tin cậy để ứng dụng vào các thuật toán phát hiện dị trường trên ảnh UAV.

Trên các không gian màu XYZ, HSL, HSB hiệu suất phát hiện dị thường của các thuật toán không ổn định, vì vậy nên cẩn trọng khi áp dụng chúng.

Các không gian màu YcbCr và YUV giường như có sự tương đồng, mặc dù có sự sụt giảm nhẹ về hiệu suất trong các thuật toán $\mathrm{CRX}$ và $\mathrm{RX}-\mathrm{UTD}$ nhưng đây là hai không gian màu cũng đáng tin cậy để được sử dụng.

Không gian màu cuối cùng trong danh sách đánh giá là CMYK, không gian màu này cho hiệu năng tốt nhất khi sử dụng thuật toán UTD, CRX đối với ảnh nền khu vực đồng bằng; cho hiệu suất tốt nhất khi sử dụng thuật toán NRX, UTD, CRX đối với ảnh nền khu vực rừng núi; cho hiệu suất tốt nhất khi sử dụng thuật toán LRX, NRX, MRX, WRX và $C R X$ đối với ảnh nền khu vực biển. Tuy nhiên, không gian màu CMYK lại là không giam màu cho hiệu suất kém nhất khi thực hiện thuật toán RX-UTD trên cả hai ảnh nền khu vực đồng bằng và rừng núi.

\section{KẾT LUẬN}

Trong công tác tìm kiếm cứu nạn, việc nâng cao hiệu suất phát hiện dấu hiệu dị thường mang ý nghĩa quan trọng, rút ngắn thời gian và phí tổn tài chính, sức lực, tinh thần, đồng thời nâng cao cơ hội cứu nạn. Kết quả khảo sát đánh giá các kỹ thuật phát hiện dị thường trên ảnh UAV thực hiện trong nghiên cứu này đã được tiếp cận khoa học dựa trên sự kết hợp của toán tử phát hiện dị thường $\mathrm{RX}$ và một số biến thề của $\mathrm{RX}$ với các không gian màu khác nhau.

Kết quả thực nghiệm trên các bộ dữ liệu mẫu cho thấy rằng trong các tình huống tìm kiếm cứu nạn khác nhau có sử dụng ảnh UAV, một không gian màu phù hợp cùng với một thuật toán phát hiện có thể được chọn để cho hiệu suất phát hiện dị thường tốt nhất. Kết quả ban đầu này của nhóm nghiên cứu sẽ từng bước được kiểm nghiệm trên các loại dữ liệu ảnh vệ tinh quang học trong các nghiên cứu tiếp theo để phát hiện đối tượng trôi dạt trên biển. Các mô hình cải biên của bộ phát hiện $R X$ và giải pháp song song trên tập dữ liệu lớn cũng sẽ được khảo sát trong các nghiên cứu tiếp theo.

\section{LờI CẢM ƠN}

Nghiên cứu này được tài trợ kinh phí từ đề tài nghiên cứu cứu khoa học cấp quốc gia mã số VT-UD.04/16-20 thuộc Chương trình Khoa học Công nghệ vũ trụ. Nhóm tác giả cảm ơn sự ủng hộ và đồng hành của Ban chủ nhiệm Chương trình.

\section{TÀI LIÊU THAM KHẢO}

[1] D. Manolakis, D. Marden, and G. A. Shaw, "Hyperspectral image processing for automatic target detection applications," Lincoln Laboratory Journal, vol. 14, no. 1, pp. 79-116, 2003.

[2] T. E. Smetek and K. W. Bauer, "Finding hyperspectral anomalies using multivariate outlier detection," in Proceedings of the Aerospace Conference, 2007.

[3] I. S. Reed and X. Yu, "Adaptive Multiple-Band CFAR Detection of an Optical Pattern with Unknown Spectral Distribution," IEEE Transactions on Acoustics, Speech, and Signal Processing, vol. 38, no. 10, pp. 1760-1770, 1990.

[4] D. C. Borghys, V. Achard, S. R. Rotman, N. Gorelik, C. Perneel, Scwheicher, and Emile, "Hyperspectral anomaly detection: a comparative evaluation of methods," in Proceedings of the General Assembly and Scientific Symposium, XXXth URSI, Feb 2011, pp. 1-4.

[5] J. C. Harsanyi, Detection and classification of subpixel spectral signatures in hyperspectral image sequences. Ph.D. dissertation, Univ. Maryland, Baltimore County, 1993.

[6] C.-I. Chang and S.-S. Chiang, "Anomaly detection and classification for hyperspectral imagery," IEEE Transactions on Geoscience and Remote Sensing, vol. 40, no. 6, pp. 13141325, 2002.

[7] H. Ren, C. Chen, and H. Chen, "Weighted anomaly detection for hyperspectral remotely sensed images," in Proceedings of the Chemical and Biological Standoff Detection III, Nov 2005.

[8] N. D. Rasmussen, D. R. Thornton, and B. S. Morse, "Enhancement of unusual color in aerial video sequences for assisting wilderness search and rescue," in Proceedings of the 15th IEEE International Conference on Image Processing, 2008, pp. 1356-1359.

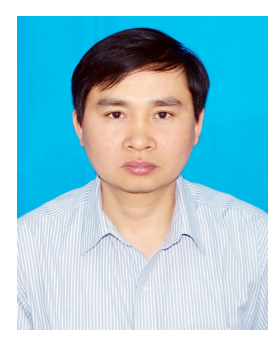

Nguyễn Văn Phương nhận bằng Kỹ sư và Thạc sĩ tại Học viện Kỹ thuật Quân sự vào các năm 2003 và 2009 . Hiện nay, tác giả là nghiên cứu sinh tại Khoa Công nghệ Thông tin, Học viện Kỹ thuật Quân sự. Lĩnh vực nghiên cứu của tác giả là GIS và xử lý ảnh viễn thám quang học.

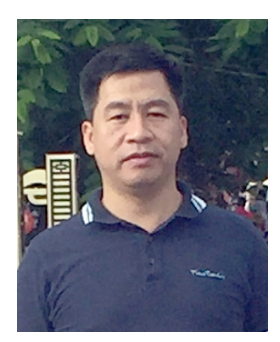

Đào Khánh Hoài nhận học vị Tiến sĩ năm 2005. Hiện nay, tác giả đang công tác tại Học viện Kỹ thuật Quân sự. Lĩnh vực nghiên cứu của tác giả là GIS, xử lý ảnh vệ tinh, $\mathrm{UAV}$, đo ảnh và thị giác máy tính. 